\title{
QUESTIONÁRIO DE DOR McGILL: PROPOSTA DE ADAPTAÇÃO PARA A LÍNGUA PORTUGUESA*
}

\author{
Cibele Andrucioli de Mattos Pimenta** \\ Manoel Jacobsen Teixeira***
}

PIMENTA, C. A de M.; TEIXEIRA, M. J. Questionário de dor McGill: proposta de adaptação para a lingua portuguesa. Rev.Esc.Enf.USP, v.30, n.3, p. 473-83, dez. 1996.

Dor é uma experiência multidimensional e subjetiva. O desenvolvimento de instrumentos que possibilitem a avaliação da dor nos seus diversos aspectos, é fundamental para a compreensão do quadro álgico, implementação da terapêtica $e$ apreciação de sua eficácia. O questionário McGill é considerado um dos melhores instrumentos para a avaliação das dimensões sensitiva-discriminativa, afetivamotivacional e cognitiva-avaliativa da dor. Apresentar proposta de sua adaptação para a lingua portuguesa é o objetivo deste estudo.

UNITERMOS: Dor. Dor crônica. Avaliação da dor. Inventário de dor. Mensuração da dor; Questionário de dor McGill.

\section{INTRODUÇÃO}

A comunicação da experiência dolorosa pelos doentes aos profissionais de. saúde que os atendem é fundamental para a compreensão do quadro álgico. implementação de medidas analgésicas e avaliação da eficácia terapêutica. Dor é um fenômeno individual e subjetivo. A necessidade de se conhecer e comparar quadros dolorosos entre populações diferentes e de quantificar a resposta às diversas terapias despertou, nos pesquisadores, o interesse em desenvolver instrumentos de avaliação de dor passíveis de comparação e que possibilitassem o desenvolvimento de uma linguagem universal sobre a experiência dolorosa.

\footnotetext{
* Trabalho concorrente ao prêmio Prof. Sérgio Ferreira (Incentivo à pesquisa sobre dor no Brasil), promovido pela Sociedade Brasileira de Estudo da Dor, 1996 e classificado em $10^{\circ}$ lugar.

* Enfermeira. Professora Doutora do Departamento de Enfermagem Médico-Cirúrgica da Escola de Enfermagem da Universidade de São Paulo. Coordenadora de Enfermagem da Liga de Dor do HC-FMUSP.

*** Neurocirurgiaxo. Professor Doutor do Departamento de Neurologia da Faculdade de Medicina da Universidade de São Paulo. Chefe do Ambulatório de Dor da Clínica Neurológica do HCFMUSP
} 
Os instrumentos para a avaliação da dor crônica em adultos baseiam-se fundamentalmente no auto-relato ${ }^{2}$. () doente é autoridade sobre sua dor, visto o caráter individual e subjetivo da experiência dolorosa.

O conceito de dor como um fenômeno diretamente relacionado d̀ extensão da lesão tecidual foi preponderante até a década de 60. Esta é a razão pela qual os primeiros trabalhos sobre avaliação da dor mediam, exclusivamente, sua intensidade. Foram elaboradas várias escalas para mensurar a intensidade da dor, mas poucas aferem aspectos sensitivos e afetivos da experiência dolorosa ${ }^{3,5}$, 6. 12. Dentre as escalas unidimensionais. destacaram-se as numéricas, onde o doente gradua a dor em intervalos de 0 a 5 ou 0 a 10 . onde 0 significa ausência de dor e 5 ou 10, respectivamente, significam a pior dor imaginável e a escala visual analogica, que consiste em uma reta de $10 \mathrm{~cm}$ onde nas extremidades constam as palavras âncoras: sem dor e pior dor imaginávele ${ }^{2,6}$. Após a publicação do trabalho de MELZACK: TORGERSON ${ }^{15}$ que enfatizou a importância das 3 dimensões da dor: a sensorial-discriminativa. a motivacional-afetiva e a cognitivaavaliativa, sustentadas por sistemas fisiologicamente especializados no SNC, foi desenvolvida a primeira escala multidimensional de avaliação da dor ${ }^{12}$. A partir da compreensão da necessidade de escalas que mensurassem as diferentes qualidades da dor, MELZACK"2 desenvolveu o "Questionário para Dor McGill ". E o instrumento mais utilizado para se avaliar outras características da dor, além da intensidade. Foi elaborado para fornecer medidas quantitativas da dor, que pudessem ser tratadas estatisticamente e permitir comunicação das qualidades sensoriais. afetivas e avaliativas do fenômeno doloroso. Tem índices de validade e confiabilidade estabelecidos e poder discriminativo entre os diversos componentes da dor ${ }^{12}$.

A dimensão sensorial-discriminativa 6 influenciada, primariamente, pelos sistemas espinais de condução rápida; a dimensão motivacional-afetiva é processada pelas estruturas da formação reticular do tronco encefálico e límbicas, que sofrem influência dos sistemas nociceptivos de condução espinal lenta. As unidades neocorticais comparam a informação nociceptiva com as experiências passadas e exercem controle sobre as estruturas responsáveis pela dimensão sensitivo- discriminativa e afetivo-motivacional. Da interação destes aspectos resulta a informação localizada têmporo-espacialmente, quantificada e qualificada. Resulta, ainda, a tendência motivacional direcionada à fuga, defesa, retirada ou ataque e a modificação do afeto. A interpretação da informação processada por unidades cognitivas é condicionada pelas experiências prévias e pode gerar respostas diferentes à experiência dolorosa em diferentes indivíduos e em diferentes momentos do mesmo individuo ${ }^{12,13.15 .16}$. Fica claro, que medir a intensidade da dor é apenas um aspecto do problema. Limitar a avaliação da experiência dolorosa apenas a intensidade é como avaliar a experiência visual apenas em termos de intensidade luminosa, esquecendo que outros elementos como cor e textura também compõem a percepção visual. A avaliação da dor visa a aferir qualidade, duração e impacto na esfera psico-afetiva, além de determinar sua intensidade. Tem a finalidade de auxiliar no diagnóstico, ajudar na escolha da terapia e quantificar a efetividade da terapêutica implementada ${ }^{12,14}$. 
O questionário McGill foi adaptado para diferentes línguas e utilizado em mais de 100 pesquisas sobre dor ${ }^{14}$. Não se conhece publicação sobre sua adaptação para a língua portuguesa. A ausência de instrumentos multidimensionais para a avaliação de dor constitui limitação para as atividades clínicas e de pesquisa sobre dor em nosso meio e motivou a elaboração deste estudo, que teve como objetivos:

- apresentar o caminho metodológico utilizado para a adaptação do questionário de dor McGill em língua portuguesa:

- verificar a opinião de doentes com dor crônica sobre a dificuldade para o preenchimento do questionário de dor McGill e de sua utilidade para descrever dor.

\section{METODOLOGIA}

Para a adaptação do questionário Mc(iill foram assumidos os seguintes pressupostos:

- por tratar-se de escala com índices de validade e confiabilidade já reconhecidos, julgou-se suficiente validar a tradução;

- validar a tradução, o que foi feito por comitê de especialistas, consistiu em assumir que os termos escolhidos em língua portuguesa mantivessem correspondência adequada aos da língua original (inglesa) e ao mesmo tempo, que fossem palavras conhecidas, de uso freqüente no cotidiano;

- as palavras de cada subgrupo deveriam manter ordem sutil de intensidade crescente.

A adaptação do questionário foi feita em várias etapas:

- $\quad 1^{a}$ fase - Tradução dos termos

Tradução do original por profissionais envolvidos no estudo da língua inglesa e portuguesa;

- $\quad 2^{a}$ fase - Validação da tradução - Comitê de especialistas

Apresentação desta proposta a um comitê composto por 5 especialistas. Foram considerados especialistas na área, profissionais com reconhecida experiência em serviços de dor. O comitê foi composto por 2 neurocirurgiões, 1 fisiatra, 1 reumatologista e 1 psicoterapêta. Estes profissionais, individualmente, opinaram sobre a tradução proposta para cada descritor e apresentaram sugestões. Foram considerados como tendo tradução adequada os descritores aceitos por, pelo menos, $80 \%$ dos especialistas. Após a compilação dos descritores, cuja tradução não fora validada por $80 \%$ dos especialistas e das sugestões apresentadas, realizaram-se 2 reuniões para discussão e adaptação dos termos restantes. O critério para aprovação da tradução foi o mesmo: $80 \%$ de concordância. 
- $\quad 3^{a}$ fase - Aplicação clínica

Obtido o consenso entre os especialistas iniciou-se a fase de aplicar o questionário em doentes com dor crônica para identificar os descritores por eles desconhecidos. O questionário de McGill inicialmente proposto foi utilizado em 57 doentes com dor crônica. ()s descritores desconhecidos por, no mínimo, 15\% dos doentes retornaram ao comitê de especialistas para nova adaptação. Após isto, a nova versão do questionário foi aplicada em 81 doentes com dor crônica e foram identificados os termos desconhecidos por no mínimo $15 \%$ dos indivíduos, que foram encaminhados ao comitê de especialistas para modificação.

Avaliação quanto ao valor e dificuldade para seu uso foi realizada através de entrevista com os doentes.

\section{RESULTADOS}

Após a tradução, foram. inicialmente, aprovados pelo comitê de especialistas $26(33,3 \%)$ dos 78 descritores. $\Lambda$ pós a compilação dos descritores cuja tradução não fora validada por $80 \%$ dos especialistas e das sugestões apresentadas, realizaram-se 2 reuniões para discussão e adaptação dos termos restantes. Após isto, todos os descritores foram aceitos por, no mínimo, 80,0\% dos especialistas. () questionário obtido inicialmente está apresentado no QUADRO 1.

QUADRO 1 - Proposta inicial de adaptação do Questionário de dor MeGill para a língua portuguesa (fases 1 e 2). São Paulo, 1995.

\begin{tabular}{|c|c|c|c|c|}
\hline 1 & 5 & 9 & 13 & 17 \\
\hline 1-ondulante & 1-fisgada & 1-vaga & 1-amedrontada & 1-esparrama \\
\hline 2-tremulante & 2-aperto & 2-dolorimento & 2-apavorante & 2-irradia \\
\hline 3-pulsante & 3-mordida & 3-machucada & 3-aterrorizante & 3-penetra \\
\hline 4-palpitante & 4-cólica & 4-dolorida & & 4-transfixa \\
\hline 5-latejante & 5-esmagamento & 5-em peso & 14 & \\
\hline \multirow[t]{2}{*}{ 6-em pancada } & & & 1-castigante & 18 \\
\hline & 6 & 10 & 2-atormenta & 1-aperta \\
\hline 2 & 1-puxão & 1-sensível & 3-cruel & 2-adormece \\
\hline 1-pontada & 2-estiramento & 2-distendida & 4-maldita & 3-repuxa \\
\hline $\begin{array}{l}\text { 2-choque } \\
\text { 3-tiro }\end{array}$ & 3-arrancamento & $\begin{array}{l}\text { 3-esfolante } \\
\text { 4-rompendo }\end{array}$ & 5-mortificante & $\begin{array}{l}\text { 4-espreme } \\
\text { 5-rasga }\end{array}$ \\
\hline 3-tiro & 7 & & 15 & \\
\hline 3 & 1-calor & 11 & 1-miserável & 19 \\
\hline 1-alfinetada & 2-queimor & l-cansativa & 2-alucinante & 1-fria \\
\hline 2-perfurante & 3-escaldante & 2-exaustiva & & 2-gelada \\
\hline 3-facada & 4-causticante & & 16 & 3-congelante \\
\hline 4-punhalada & & 12 & 1-maçante & \\
\hline \multirow[t]{2}{*}{ 5-lancinante } & 8 & 1-enjoada & 2-incômoda & 20 \\
\hline & 1-formigamento & 2 -sufocante & 3-desgastante & 1-aborrecida \\
\hline 4 & 2-coceira & & 4-intensa & 2-nauseante \\
\hline 1-aguda & 3-ardor & & 5-insuportável & 3-agonizante \\
\hline 2-cortante & 4-ferroada & & & 4-pavorosa \\
\hline 3-dilacerante & & & & 5-torturante \\
\hline
\end{tabular}


Após a etapa desenvolvida pelo comitê de especialistas, iniciou-se a aplicação do qüestionário McGill em doentes com dor crônica (fase 3). Observouse que significativa parcela dos descritores de dor não era compreendida pelos indivíduos. Na primeira aplicação clínica, que envolveu 57 doentes, encontrouse que $21,8 \%$ dos descritores eram desconhecidos por, no mínimo, $15 \%$ dos doentes. Estes descritores foram ajustados pelo comitê de especialistas e submetidos a uma segunda aplicação clínica, que envolveu 81 doentes com dor crônica. Cabe ressaltar que neste momento, somente $3(3,8 \%)$ descritores precisaram ser adaptados.

Os 57 doentes com dor crônica de origem oncologica que participaram da primeira avaliação clínica do questionário Me( iill eram em 50,9\% dos casos homens, possuíam idade média de 50,1 anos. escolaridade média de 4,9 anos e renda per capita mensal média de 1,4 salário mínimo. Os 81 doentes com dor crônica de etiologia variada (cefaléia, distrofia simpático-reflexa, entre outras), que participaram da segunda avaliação clínica eram homens em $23,7 \%$ das vezes, possuíam idade média de 49,1 anos, escolaridade média de 5,7 anos e renda per capita mensal média de 2 salários mínimos.

() questionário obtido, após a aplicação clínica, está apresentado no QUADRO 2.

QUADRO 2 - Proposta de adaptação do Questionário de dor de MeGILL para a língua portuguesa. São Paulo, 1995.

ALGUMAS PALAVRAS QUE EU VOU LER DESCREVEM A SUA DOR ATUAL. DIGA-ME QUAIS PALAVRAS MELHOR DESCREVEM A SUA DOR. NÃO ESCOLHA AQUELAS QUE NÃO SE APLICAM. ESCOLHA SOMENTE UMA PALAVRA DE CADA GRUPO. A MAIS ADEQUADA PARA A DESCRIÇÃO DE SUA DOR.

\begin{tabular}{|c|c|c|c|c|}
\hline 1 & 5 & 9 & 13 & 17 \\
\hline 1-vibraçăo & 1-beliscăo & 1.-mal localizada & 1-amedrontadora & 1-espalha \\
\hline 2-tremor & 2-aperto & 2-dolorida & 2-apavorante & 2-irradia \\
\hline 3-pulsante & 3-mordida & 3-inachucada & 3-aterrorizante & 3-penetra \\
\hline 4-latejante & 4-cólica & 4-doída & & 4-atravessa \\
\hline 5-como batida & 5-esmagamento & 5-pesada & 14 & \\
\hline \multirow[t]{2}{*}{ 6-como pancada } & & & 1-castigante & 18 \\
\hline & 6 & 10 & 2-atormenta & 1-aperta \\
\hline 2 & 1-fisgada & 1-sensivel & 3-cruel & 2-adormece \\
\hline 1-pontada & 2-puxăo & 2-esticada & 4-maldita & 3-repuxa \\
\hline 2-choque & 3-em torçăo & 3-esfolante & 5-mortal & 4-espreme \\
\hline \multirow[t]{2}{*}{ 3-tiro } & & 4-rachando & & 5-rasga \\
\hline & 7 & & 15 & \\
\hline 3 & 1-calor & 11 & 1-miserável & 19 \\
\hline 1-agulhada & 2-queimaçăo & 1-cansativa & 2-enlouquecedora & 1-fria \\
\hline 2-perfurante & 3-fervente & 2-exaustiva & & 2-gelada \\
\hline 3-facada & 4-em brasa & & 16 & 3-congelante \\
\hline 4-punhalada & & 12 & 1-chata & \\
\hline \multirow[t]{2}{*}{ 5-em lança } & 8 & 1-enjoada & 2-que incomoda & 20 \\
\hline & 1-formigamento & 2-sufocante & 3-desgastante & 1-aborrecida \\
\hline 4 & 2-coceira & & 4 -forte & 2-dá náusea \\
\hline 1-fina & 3-ardor & & 5-insuportável & 3-agonizante \\
\hline 2-cortante & 4-ferroada & & & 4-pavorosa \\
\hline 3-estraçalha & & & & 5-torturante \\
\hline
\end{tabular}




\begin{tabular}{|c|c|}
\hline Número de Descritores & Índice de Dor \\
\hline $\begin{array}{l}\text { Sensorial.............. } \\
\text { Afetivo.................. } \\
\text { Avaliativo............. } \\
\text { Miscelânea.......... } \\
\text { TOTAL.............. }\end{array}$ & 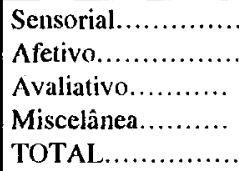 \\
\hline
\end{tabular}

\section{LEGENDA}

Os sub-grupos de 1 a 10 representam respostas sensitivas à experiência dolorosa (tração, calor, torção, entre outros); as descritores dos sub-grupos de 11 a 15 são respostas de caráter afetivo (medo, punição, respostas neurovegetativas, etc.); o sub-grupo 16 e avaliativo (avaliação da experiência global) e os de 17 a 20 são miscelânea.

O tempo necessário para o preenchimento do qüestionário variou entre 3 e 4 minutos.

Quando questionados quanto ao grau de dificuldade para preenchimento do inventário e sobre a utilidade do questionário para explicar a dor, entre os 81 doentes avaliados, apenas $3,7 \%$ considerou-o difícil ou muito dificil de preencher. Quanto ao auxílio que o questionário forneceu para a descrição de seu quadro doloroso, observou-se que $96 \%$ escolheu as categorias "aju dou muito" e "ajudou".

\section{DISCUSSÃO}

A dor apresenta qualidades diferentes, de acordo o orgão acometido, natureza do agente lesivo e características indivíduais. Sabe-se que a expressâo cólica significa dor visceral e queimor, lesão de nervos periféricos. Estas expressões fornecem a chave do diagnostico e sugerem a terapia antiálgica. No entanto, a vivência de dor intensa o freqüentemente acompanhada de dificuldade para descrevê-la. () questionário de dor de Mc(iill é considerado o melhor instrumento e 6 o mais utilizado para caracterizar e discernir os componentes afetivo, sensitivo e avaliativo da dor, quando se pretende obter informações qualitativas e quantitativas a partir de descrições verbais. É considerado instrumento universal, capaz de padronizar a linguagem da dor ${ }^{14}$. Foi elaborado a partir do levantamento, em literatura especializada, de 102 palavras utilizadas para descrever dor. Profissionais de saúde universitários organizaram as palavras, consideradas qualitativamente semelhantes, em 3 grupos: sensorial, afetivo e avaliativo. Algumas dessas palavras (descritores) são sinônimos entre si, outras parecem sinônimos, mas variam em intensidade, enquanto outras apresentam diferenças mínimas ou nuances que podem ser importantes para as 
pessoas que estão tentando comunicar sua dor. Em um segundo momento, organizaram os descritores em 16 subgrupos, de acordo com o tipo de sensação que descreviam. Dentro de cada subgrupo, os descritores foram organizados em graus (seqüência crescente de intensidade). Como algumas pessoas sentiam falta de algumas palavras, foi acrescido um $4^{\mathrm{o}}$ grupo (composto por 4 subgrupos), chamado de miscelânea. Na apresentação final, o questionário foi constituído por 4 grupas (sensitivo-descriminativo, afetivo-motivacional, cognitivo-avaliativo e miscelânea), 20 subgrupos e 78 descritores ${ }^{12}$.

O grupo sensorial-discriminativo (subgrupos de 1 a 10) refere-se às propriedades mecânicas, térmicas, de vividez e espaciais da dor; o grupo afetivomotivacional (subgrupos de 11 a 15) descreve a dimensão afetiva nos aspectos de tensão, medo e respostas neurovegetativas; os descritores do componente cognitivo-avaliativo (subgrupo 16) permitem, ao doente, expressar a avaliação global da experiência dolorosa. ()s subgrupos de 17 a 20 compreendem ítens de miscelânea. Cada subgrupo é composto por 2 a 6 descritores qualitativamente similares, mas com nuances que os tornam diferentes em termos de magnitude. Assim, para cada descritor corresponde um número que indica sua intensidade.

A partir do questionário de Mc(ill, pode-se chegar às seguintes medidas: número de descritores escolhidos e índice de dor. (O) número de descritores escolhidos corresponde as palavras que o doente escolheu para explicar a dor. $O$ maior valor possível e 20 , pois o doente só pode escolher, no máximo, uma palavra por subgrupo. (O índice de dor é obtido através da somatória dos valores de intensidade dos descritores escolhidos. () valor máximo possível 6 . 78. Estes índices podem ser obtidos no total e para cada 1 dos 4 componentes do questionário: padrão sensitivo, afetivo, avaliativo e subgrupo de miscelânea.

O questionário de Mc(aill contém ainda uma escala de intensidade (0 a 5), um diagrama corporal para representação do local da dor e a caracterização de aspectos como periodicidade e duração da queixa álgica

O reconhecimento de que este questionário é o melhor instrumento existente para se avaliar a dor sob o prisma multidimensional, a compreensão de que sua elaboração foi calcada no referencial tébrico da fisiologia da dor ${ }^{3,15}, 0$ que pressupõe alguma universalidade das qualidades álgicas e, finalmente, os estudos $^{3,7}$, que confirmaram que indivíduos com diferentes antecedentes socioculturais, mas com sintomas similares, tendem a escolher as mesmas palavras para descrever sua experiência dolorosa, estimularam a adaptação do questionário para diferentes línguas tais como: italiano, espanhol, francês, dinamarquês, alemão e finlandês ${ }^{3.8 .9 .10,11.14}$

Traduzir e validar a tradução de instrumento do tipo McGill é tarefa delicada e demorada, pois é preciso manter correspondência com a estrutura teórica original do inventário e, ao mesmo tempo, torná-lo compreensível aos doentes. Se o significado do descritor ou sua intensidade diferem da estrutura original, o objetivo de se ter um instrumento universal para avaliar dor fica comprometido. No presente estudo, considerou-se que, inicialmente, a validação seria feita por especialistas na área de dor. A seguir o inventário foi aplicado em 
doentes com dor, para adequação dos descritores cujo significado fosse desconhecido por, no mínim. 15\% dos avaliados. Acredita-se que o procedimento de submeter o questionário à avaliação dos doentes permitiu o aperfeicoamento da proposta de tradução.

Para adaptar o questionário McGill nas diferentes línguas diversas técnicas foram utilizadas ${ }^{1}$. A tradução dos descritores de dor, originalmente em língua inglesa, para a língua desojada o uma das possibilidades e apresenta como vantagem o possível paralelismo com o inventário original, o que permite que estudos sobre dor desenvolvidos em diferentes culturas. possam ser comparados. No entanto, a tradução pura e simples pode resultar em um questionário com termos não usuais no cotidiano, que poderiam ser rejeitados ou não compreendidas pelos doentes. () procedimento intermediário consiste em traduzir parte dos descritores e introduzir. em parte. novos descitores nacionais. Uma outra possibilidade 6 a construção da escala na lingua desejada. seguindo-se os passos utilizados na construção do Mc(iill. $\Lambda$ vantagem e a introdução de termos nacionais e a desvantagem o que de tal procedimento pode resultar um questionário diferente, o que limita comparações transculturais, além da complexidade de construção e validação desta "nova" escala.

MAIANI: SANAVIO" traduziram os descritores para a língua italiana e solicitaram a 34 indivíduos sem dor que atriluuíssem valor de intensidade a cada descritor. Compararam a média de intensidade de cada descritor à média dos descritores descrita por Molzack. ()s descritores cujas médias diferiram significativamente nesta comparação foram analisados por um grupo de indivíduos bilíngues, que atribuíram novos valores de intensidade a cada descritor. A seguir. os descritores foram organizados nos subgrupos a partir da média de intensidade obtida. KISS et al. " optaram por realizar a tradução dos termos ingleses para o alemão. com a ajuda do professores de língua inglesa. Também LAHUER'TA et al. ${ }^{10}$ realizaram tradução literal do inventário para a língua espanhola. KETOVU()Rl: PöNTINEN fizeram tradução dos descritores de dor do questionário de Mc(xill para o finlandôs e os organizaram om ordem alfabética. Solicitaram a estudantes o doentes que agrupassem descritores semelhantes e os alocassem. individualmente. na escala visual analógica de intensidade de dor. Outro grupo do 76 estudantes univorsitários julgou se as palavras nos subgrupos expressavam qualidades similares. Foram aceitas as palavras avaliadas como adequadamente alocadas por, no mínimo, $50 \%$ dos juízes. A partir do valor médio de intensidade, os descritores foram organizados dentro de cada grupo. DREWES et al. ${ }^{2}$ utilizaram melologia semelhante a utilizada pelos pesquisadores finlandeses para elaborar a versão do Mc(iill para o dinamarquês. BENEDITTSS et. al. ' refizeram a trajetória u tilizada por MEIZACK para a construção do McGill. Se]ecionaram 203 palavras relativas à dor existentes na literatura italiana. Estudantes e médicos agruparam as palavras semelhantes e as organizaram em ordem crescente de intensidade. Obtiveram questionário com 42 descritores de dor distribuídos em 3 classes (sensorial, afetiva e avaliativa) e 16 subgrupos. 
Os procedimentos utilizados neste trabalho para desenvolver a versão em língua portuguesa, como tradução com ajuda de profissionais da língua inglesa e utilização de juízes, assemelham-se aos utilizados em outros trabalhos. As sucessivas avaliações do inventário pelos doentes e retorno aos juízes para adaptação dos termos não foi utilizada por outros autores. Há ainda etapas a serem desenvolvidas como a comprovação de que os descritores estão organizados em ordem crescente dentro de cada subgrupo e testes clínicos. elulga-se que a validação da adaptação do questionário deva incluir etapa de aplicação do inventário em doentes com diferentes síndromes dolorosas para comparação dos resultados obtidos com os de outros trabalhos, para se verificar se o inventário em lingua portuguesa equivale ao original. Tal procedimento foi realizado em doentes com dor crônica (cefaléia, doença oncológica e distrofia simpático-reflexa). Esta experiência será objeto de publicação futura.

Apesar de ser reconhecido como instrumento de grande valor para a aferição de numerosos aspectos associados à dor, algumas reflexões têm sido feitas acerca do questionário McGill. É citado que os descritores são de difícil compreensão por indivíduos com baixa escolaridade, idosos ou aqueles com dificuldade de concentração. Não se encontraram estudos que tivessem avaliado a magnitude desta dificuldade. A baixa escolaridade da população brasileira acentua a preocupação com a possibilidade da adequada compreensão do questionário. No entanto, os resultados obtidos mostraram que mais de $95 \%$ dos entrevistados disse ser capaz de compreender o inventário sem grande dificuldade.

O tempo para o seu preenchimento também é apontado como limitação para seu uso clínico. Neste estudo, o tempo médio para sua aplicação foi em torno de 3 a 4 minutos, por doente. Este tempo é, sem dúvida, superior ao necessário para se aplicar uma escala de intensidade, mas a qualidade e quantidade de informações que se obtém são superiores. No entanto, talvez seja útil construir um instrumento reduzido para uso clínico diário, à semelhança do "The McGill Short-Form" ${ }^{13}$.

Quanto à forma de aplicação verbal ou escrita, GRAHAM ${ }^{4}$ não encontrou diferenças significativas nas respostas. Entretanto, para avaliar dores ocorridas há mais de 1 semana, os indivíduos sentiam dificuldade para lembrar e sumarizar sua experiência. Recomendou o uso do questionário de McGill para dores imediatas ou as ocorridas há até 1 semana, que foi o critério utilizado na presente pesquisa.

Dos 78 descritores que compõem o questionário de McGill, $42(53,9 \%)$ referem-se ao aspecto sensitivo da dor; $14(17,9 \%)$ ao componente afetivo, $5(6,4 \%)$ à avaliação da experiência dolorosa e $17(21,8 \%)$ ao subgrupo de miscelânea. Tem sido motivo de questionamentos a distribuição desproporcional dos descritores entre os 3 componentes (sensitivo, afetivo e avaliativo), acrescido do fato de que a maioria dos descritores do subgrupo miscelânea é de caráter sensitivo. Acreditase que, decorrente desta diferença de proporções, o doente pode ser impelido a escolher mais descritores sensitivos ${ }^{2}$.

Apesar dessas críticas, parece inquestionável o auxílio do inventário para o doente comunicar a sua experiência de dor. Observou-se, neste estudo, que 
acima de $95 \%$ dos doentes afirmou ter se beneficiado com o uso do questionário McGill. Algumas opiniões dos doentes sobre a utilidade do questionário estão abaixo transcritas:

“A gente não consegue explicar e a resposta já está pronta”.

"Achei muito bom porque, às vezes, não encontrava as palavras certas e o qüestionário tem as palavras certas".

"É muito difícil explicar como é a dor. Transferiu o que sinto para palavras".

\section{CONCLUSÃO}

A avaliação da dor, para fins clínicos e de pesquisa, depende da descrição verbal da experiência pessoal, não somente da intensidade mas também das qualidades da dor. O questionário de Mc(xill emergiu como um instrumento clínico e de pesquisa largamente utilizado. Novos estudos serão necessários para validar a tradução do questionário de McGill para a língua portuguesa e aquisição de experiência com o uso deste instrumento. Somente desta forma, será possível ampliar o conhecimento acerca de suas possibilidades e construir julgamento sobre sua utilidade em nosso meio. Há diversos instrumentos para a mensuração das diferentes qualidades da dor, alguns muito bem estabelecidos e outros que precisam ainda ser testados e aperfeiçoados. Implementar o que já existe, na atividade clínica e de pesquisa, é necessário.

Agradecimentos: aos Drs. Cláudio Fernandes Correa, João Augusto B. Figueiro, Lin T. Yang e Moacir Nobre, por terem atuado como especialistas. A Sra. Cristina Aoki, pela ajuda em parte da coleta dos dados.

PIMENTA, C. A. de M.; TEIXEIRA, M. J. Adaptation of McGill questionnaire to portuguese language. Rev.Esc.Enf.USP. v.30, n.3. p. 473-83, dec. 1996.

Pain is a multidimensional and subjective experience. The developing instruments for the assessment of the multiple components of pain is necessary for the compreension of the suffering, to delimate therapeutic programs and to evaluate their efficacy. The McGill pain questionnaire has been considered the best instrument to assessment the sensitive-discriminative, affective-motivational and cognitiveavaliative dimensions of pain. The aim of this study is to present the adaptation of McGill to portuguese language.

UNITERMS: Pain. Chronic pain. Pain Assessment. Pain instruments. Pain measurement, McGill Pain Questionnaire. 


\section{REFERÊNCIAS BIBLIOGRÁFICAS}

1.BENEDITIS. G. de et al. The italian pain questionnaire. Pain. v. 33, p.53-62, 1988.

2.CHAPMAM, C. R. et al. Pain measurement: an overview. Pain, v.22. p.1-31. 1985.

3.DREWES, A. M. et al. McGill pain questionnaire translated into Danish: experimental and clinical findings. Clin.J. Pain, v.9, n.2, p.80-7, 1993.

4.GRACELY, R. H.; DUBNER. R. Pain assessment in humans a reply to hall.

Pain, v.11, n.1, p.109.20. 1981

5.GRAHAM, C. Use of the McGill pain questionnaire in the assessment of cancer pain: replicability and consistency. Pain. v.8, n.3, p.377.87,1980

6.HUSKISSON, E. C.'Measurement of pain. Lancet, v.2, n.7889, p.1127-31, 1974.

7.JENSEN, M. P: KAROLY, P: BRAVER, S. The measurement of clinical pain intensity: a comparison of six methods. Pain, v.27, n.1, p.11726, 1986.

8.JOHANSSON, F, G. et al. Similarities in pain descriptions of four different ethnic-cultural groups. J.Pain Symptoin. Manage vol.5, n.2, p.94-100, 1990.

9.KETOVUORI, H.; PONTINEN. P. J. A pain vocabulary in finnish the finnish pain questionnaire. Pain, v.11, n.2, p.247-53, 1981.

10.KISS, I. et al. The McGill pain questionnaire-german version: a study on cancer pain. Pain, v.29, p.195-207, 1987.

11.LAHUERTA. J. et al. An adaptation of the McGill pain questionnaire to the spanish language. Schmerz. v.3, p.132-4. 1982.

12.MAIANI. G.; SANAVIO. E. Semantics of pain in Italy: the italian version of the McGill pain questionnaire. Pain . v.22, p.399-405, 1985.

13.MELZACK, $R$. The McGill pain questionnaire: major properties and scorin methods, Pain, v. 1, n.3, p.277-99, 1975.

14.MELZACK, R. The short-form of McGill pain questionnaire, Pain, v.30, p.191-7, 1987.

15. MELZACK, R.; KATZ, J. Pain measurement in persons in pain. In: WALL, P. D.; MELZACK, R. Textbook of Pain. 3.ed. Edinburgh, Cliurchill Livingstone, 1994, cap.18, p.337-51.

16.MELZACK, R.; TORGERSON, W. S. On the language of pain. Anesthesiology, v.34, n.1, p. $50-9,1971$.

17. TEIXEIRA, M. J. Tratamento neurocirúrgico da dor. In: RAIA, A.A.; ZERBINI, E.J. Clínica cirúrgica Alípio Correa Neto. 4ed. São Paulo, Sarvier, 1988, v.2. cap.62, p.541-72. 